INSTITUT NATIONAL DE RECHERCHE EN INFORMATIQUE ET EN AUTOMATIQUE

\title{
Shape Smoothing using Double Offsets
}

Frédéric Chazal — David Cohen-Steiner — André Lieutier — Boris Thibert

\section{$\mathbf{N}^{\circ} 5991$}

Octobre 2006

Thème SYM

\section{apport}

de recherche 



\title{
RINRIA
}

\section{Shape Smoothing using Double Offsets}

\author{
Frédéric Chazal ${ }^{*}$, David Cohen-Steiner ${ }^{\dagger}$, André Lieutier ${ }^{\ddagger}$, Boris Thibert ${ }^{\S}$ \\ Thème SYM — Systèmes symboliques \\ Projet Geometrica
}

Rapport de recherche $\mathrm{n}^{\circ} 5991$ - Octobre 2006 - 21 pages

\begin{abstract}
It has been observed for a long time that the operation consisting of offseting a solid by a quantity $r$ and then offseting its complement by $d<r$ produces, in some cases, a new solid with the same topology but with a smooth boundary. While this fact has been widely used in Computer Aided Geometric Design or in the field of image processing, we provide here for the first time a tight and robust condition that garanty the smoothness of the new solid and gives a lower bound on its reach (distance to the medial axis). This condition is based on the general properties of the distance function to a compact set and relies on the recently introduced critical function and $\mu$-reach.
\end{abstract}

Key-words: Solid Modeling, Smoothing, Distance Function, Medial Axis

* INRIA Futurs, projet Geometrica, frederic.chazal@inria.fr

$\dagger$ INRIA Sophia, projet Geometrica, david.cohen-steiner@sophia.inria.fr

‡ Dassault Systèmes (Aix-en-Provence) and LMC-IMAG, Grenoble, France, andre_lieutier@ds-fr.com

$\S$ LMC-IMAG, Grenoble France, Boris.Thibert@imag.fr

Unité de recherche INRIA Futurs

Parc Club Orsay Université, ZAC des Vignes,

4, rue Jacques Monod, 91893 ORSAY Cedex (France)

Téléphone : +33172925900-Télécopie : +33160196608 


\section{Régularisation de Solides par Dilatation/Erosion}

Résumé : Il a été observé depuis longtemps que l'opération consistant à dilater un solide d'un rayon $r$ puis à dilater son complémentaire d'un rayon $d<r$ produit, dans certain cas, un nouveau solide avec un bord lisse. Bien que ce fait ait été largement utilisé en Computer Aided Geometric Design ou en traitement d'images, nous donnons pour la première fois une condition robuste et optimale qui garantit la régularité de l'objet obtenu par dilatation et érosion. Nous donnons également un minorant de ses rayons de courbures. Nos résultats sont basés sur des propriétés générales des fonctions distances à des ensembles compacts et sont reliés à la notion récemment introduite de $\mu$-reach.

Mots-clés : Régularisation, Solid Modeling, Fonction distance, Axe médian 

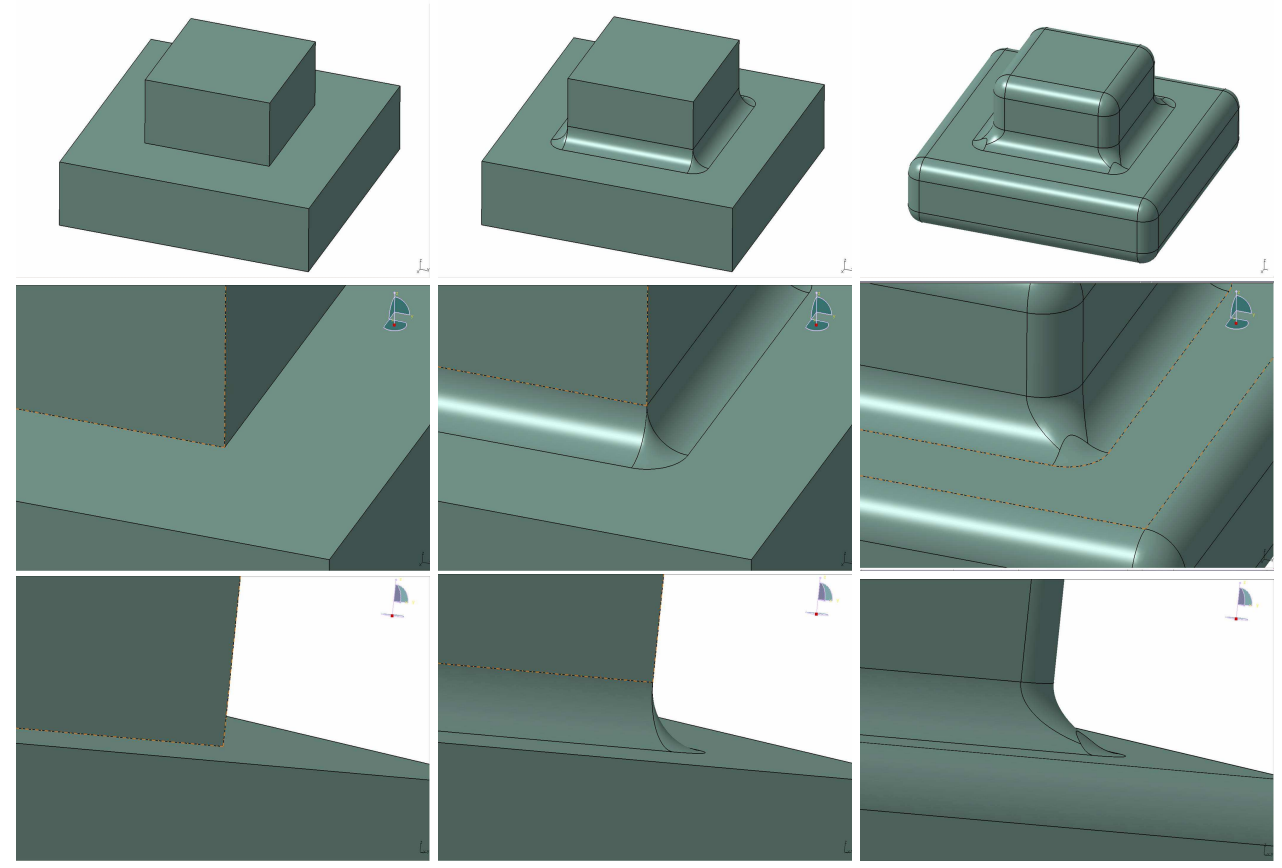

Figure 1: Two successives "rounding" by double offset illustrated on a simple CAD model

\section{Introduction}

Motivation. In Computer Aided Geometric Design (CAGD) applications, blending or filleting operators consist in "rounding" sharp edges and corners of objects. It may be motivated by aesthetic or ergonomy, constrained by manufacturing processes such as machining or moulding, or required by functional issues such as structural or aerodynamical behaviour.

A widely used geometric definition, illustrated on figure 1 consists in the so called blends by rolling ball $[24,25,26]$. Figure 1 shows on the left a simple solid, made of the union of two "boxes". In the middle, concave edges have been rounded, while, on the rigth, all edges have been rounded. Starting from the solid on the left, the rounding of concave edges can be defined by the following fictive operation. Assuming the solid to be made of a hard material (like steel) one embed it in a bloc of wax. Then one uses a spherical ball of given radius to remove as much wax as possible to obtain the solid on the middle of the figure (we reuse here a construction used by H. Edelsbrunner for the definition of $\alpha$-shapes, starting from a set of "hard points"). The remaining solid is said to be the initial one rounded from the outside by a rolling ball. The rounding of convex edges can be obtained by applying the same fictive process to the complement of the solid: one build the complement of the part in the middle out of a hard material, fill it with wax and remove (from the inside!) as much wax as possible with a spherical ball. Taking again the complement of the resulting solid yields 
the solid on the right of figure 1 . This definition of rounding is rather natural if one's intent is to design a part which have to be machined by a spherical tool (or whose mold has to be machined by a spherical tool). In many situations, this rounding process produces solids with smooth boundaries. However, in the situation of figure 2 , the center of the rolling ball follows an elliptic trajectory. When the minimum curvature radius of the ellipse is smaller than the radius of the ball, the boundary of the set swept by the ball is not smooth (see figure 2). Theorem 4.3 gives a condition determining the smoothness of such kinds of rounded solids for a large class of compact subsets of $\mathbb{R}^{n}$. Mature CAD systems, such as CATIA V5, avoid the situations where a rolling ball or an offset surface may be non smooth. These situations are detected and alternative geometric definitions are used for edges fillets. However, the issue of the robust computation of large offsets as well as a general specification for fillets operations are still open.

In order to relate the CAGD notion of rounding by rolling ball both with mathematical morphology operator and with the notion of offset used through the paper, let us introduce a few notations. The distance function $R_{K}$ of a compact subset $K$ of $\mathbb{R}^{n}$ associates to each point $x \in \mathbb{R}^{n}$ its distance to $K$ :

$$
x \mapsto R_{K}(x)=\min _{y \in K} d(x, y)
$$

where $d(x, y)$ denotes the euclidean distance between $x$ and $y$. Conversely, this function characterizes completely the compact set $K$ since $K=\left\{x \in \mathbb{R}^{n} \mid R_{K}(x)=0\right\}$.

For a positive number $r$, we denote by $K_{r}$ the $r$-offset of $K$, defined by $K_{r}=\left\{x \mid R_{K}(x) \leq r\right\}$. Given $X \subset \mathbb{R}^{n}$, one denotes by $X^{c}, \partial X$ and $\bar{X}$ respectively the complement, the boundary and the closure of $X$. In the field of image processing and mathematical morphology [22], $K_{r}$ is named the dilatation of $K$ by a ball of radius $r$, while the operation taking the complement of the dilatation of the complement, is named the erosion of $K$ by a ball of radius $r$ and is denoted $K_{\downarrow r}=\overline{\left(\left(\overline{K^{c}}\right)_{r}\right)^{c}}$. Notice that the process of "removing as much wax as possible with a ball of radius $r$ " (from left to middle on figure 1) can be expressed as $K \mapsto\left(K_{r}\right)_{\downarrow r}$, while the symmetric operation (from middle to right on figure 1) can be expressed as $K \mapsto\left(K_{\downarrow r}\right)_{r}$.

Applying both transformations (from left to right on figure 1) can thus be expressed as:

$$
K \mapsto\left(\left(\left(K_{r}\right)_{\downarrow r}\right)_{\downarrow r}\right)_{r}
$$

or, after simplification, by :

$$
K \mapsto\left(\left(K_{r}\right)_{\downarrow 2 r}\right)_{r}
$$

J. Serra and G. Matheron [22] have used erosion and dilatation operators as filters for image smoothing, removal of high frequency or topological noise. Applying successively an erosion and a dilatation remove unwanted topological features such as non significant small holes, tunnels, too thin connected components or handles. For example, in the context of medical imaging, such topological features that would be of the same order of magnitude or smaller than the accuracy of the physical measure producing the image should be considered as "topological noise" and should therefore be filtered out. This point of view of filtering is related to more recent results $[9,7,11]$, showing how simple offsets may allow to recover faithfully the topology of objects from noisy point samples or other geometric approximations. 

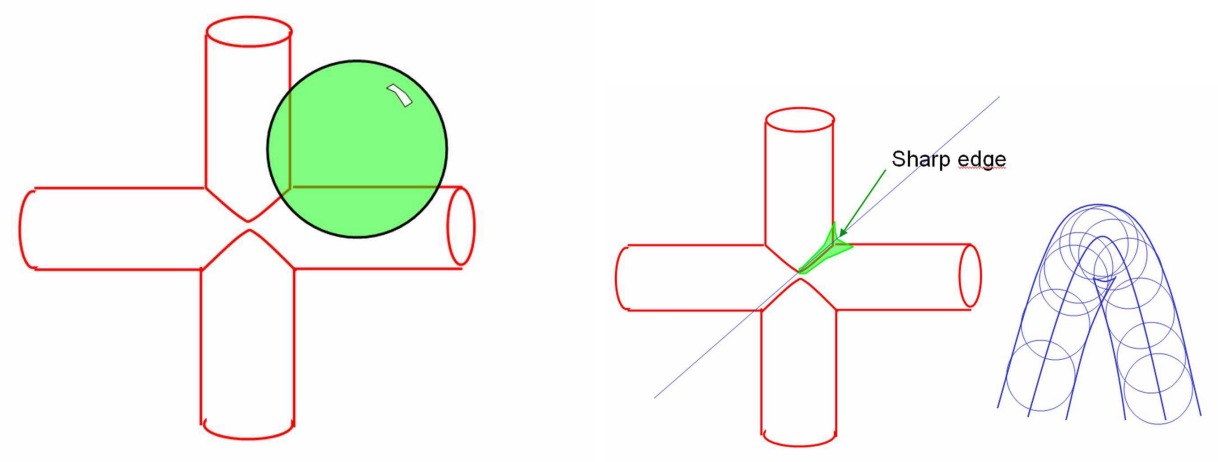

Figure 2: A situation where the hull of the rolling ball is not smooth

Let us mention still another important context in which a procedure that, starting from a compact set, could produce a nearby (nearby in some geometrical or topological sense) smooth object, could be much welcome. For the sake of robustness, it is of prime importance to know when rounding $[17,14]$ the coordinates of the vertices of a polyhedral surface on a fixed integer or floating point grid is "safe", that is avoid to break the embedding of the surface by creating unwanted "self-intersections". Indeed, here, and in the sequel of the paper, smoothness is measured through the "reach", which is the minimum distance between the object and its medial axis (see section 2.1 below). If a triangulated surface $T$ approximates a smooth surface $S$ with a sufficiently large reach, assuming some lower bounds on the size and fatness of the triangles of $T$ and on the angular accuracy of the approximation, one is able to garantee a "safe rounding" of the vertices of $T$. Similarly, it would be possible to control when a chordal error driven edge collapse procedure could preserve the embedding of $T$ (i.e. could avoid self-intersections). Garanties on the stability of the embedding is also related to the concern of the topological stability of polyhedral or Boundary representations $[2,3,1]$

Contributions. The main contributions are theorems 4.1,4.3 and 5.1 that express the smoothness, through a lower bound on the reach, of the boundary $K_{r, d}$ of $\left(K_{r}\right)_{\downarrow d}$. Combining results from Federer [16] and $\mathrm{Fu}[18]$ it has already been noted that, in the condition of theorem 4.3, the reach of $K_{r}^{c}$ is strictly positive ([5], p.22). However, beyond the trivial case where $K$ is convex, our result is the first quantitative lower bound on this reach (known from the authors). In the condition of the theorem, this bound is tight for dimension 3 and higher (see example in section 4.2).

Our lower bound relies on the notion of critical function of a compact set, introduced in [7], where it is shown that the critical function is stable (see theorem 2.3 below) and therefore computable in theory: one can derive a guaranteed bound on the reach of the double offset of an object from a partial geometric knowledge of the object, for example a Hausdorff approximation given from a measure or a sampling of the object. 
Outline. Section 2 gives some definitions used in the sequel of the paper and briefly introduce some properties of the distance function and its generalized gradient, as well as the central notions of critical function and $\mu$-reach. Section 3 gives some results on the Hausdorff distance to offset boundaries used in the proof of subsequent sections. Section 4 gives the main results of the paper, namely lower bounds on the reach of complements of offsets in theorems 4.1 and 4.3. Section 5 complements section 4 with some results on the smoothness and topological stability of double offset boundaries. In particular a guaranty on the topology of offset boundary (theorem 5.4) is given in the special case where the initial compact set is a surface in $\mathbb{R}^{3}$. Section 6 concludes by sketching some possible extensions of this work.

Acknowledgement The authors were partially supported by ANR under grant JC05_41922 "GeoTopAl: Topologie, Géométrie Différentielle et Algorithmes". The tight example of section 4.2 is an adaptation of an example suggested by J. Rossignac.

\section{Definitions and background on Distance Functions}

We are using the following notations in the sequel of the paper. Given $X \subset \mathbb{R}^{n}$, one denotes by $X^{c}$ the complement of $X$, by $\bar{X}$ its closure and by $\partial X$ the boundary of $X$. Given $A \subset \mathbb{R}^{n}, \operatorname{co}(A)$ denotes the convex hull of $A$.

The distance function $R_{K}$ of a compact subset $K$ of $\mathbb{R}^{n}$ associates to each point $x \in \mathbb{R}^{n}$ its distance to $K$ :

$$
x \mapsto R_{K}(x)=\min _{y \in K} d(x, y)
$$

where $d(x, y)$ denotes the euclidean distance between $x$ and $y$. Conversely, this function characterizes completely the compact set $K$ since $K=\left\{x \in \mathbb{R}^{n} \mid R_{K}(x)=0\right\}$. Note that $R_{K}$ is 1-Lipschitz. For a positive number $r$, we denote by $K_{r}$ the $r$-offset of $K$, defined by $K_{r}=\left\{x \mid R_{K}(x) \leq r\right\}$. The Hausdorff distance $d_{H}\left(K, K^{\prime}\right)$ between two compact sets $K$ and $K^{\prime}$ in $\mathbb{R}^{n}$ is the minimum number $r$ such that $K \subset K_{r}^{\prime}$ and $K^{\prime} \subset K_{r}$. It is not difficult to check that the Hausdorff distance between two compact sets is the maximum difference between the distance functions associated with the compact sets:

$$
d_{H}\left(K, K^{\prime}\right)=\sup _{x \in \mathbb{R}^{n}}\left|R_{K}(x)-R_{K^{\prime}}(x)\right|
$$

Given a compact subset $K$ of $\mathbb{R}^{n}$, the medial axis $\mathcal{M}(K)$ of $K$ is the set of points in $\mathbb{R}^{n} \backslash K$ who have at least two closest points on $K$. The minimal distance between $K$ and $\mathcal{M}(K)$ is called, according to Federer, the reach of $K$ and is denoted reach $(\mathrm{K})$. $\operatorname{reach}(\mathrm{K})=0$ if $K$ has sharp edges or corners. A $\mathcal{C}^{1,1}$ function is a $\mathcal{C}^{1}$ function whose first derivative is Lipschitz. A $\mathcal{C}^{1,1}$ hypersurface $S$ is a $(d-1)$-manifold embedded in $\mathbb{R}^{d}$ such that each point of $S$ has a neihborhood which is the regular image ( that is the image by a function whose derivative has maximal rank) by an injective $\mathcal{C}^{1,1}$ function of a neihborhod of 0 in $\mathbb{R}^{d-1}$. Informally, one can say that a $\mathcal{C}^{1,1}$ surface is a surface with bounded curvature, which is stricly stronger than $C^{1}$ and stricly weaker than $C^{2}$. An embedded $\mathcal{C}^{1}$ compact manifold is $\mathcal{C}^{1,1}$ if and only it has positive reach (Federer). 


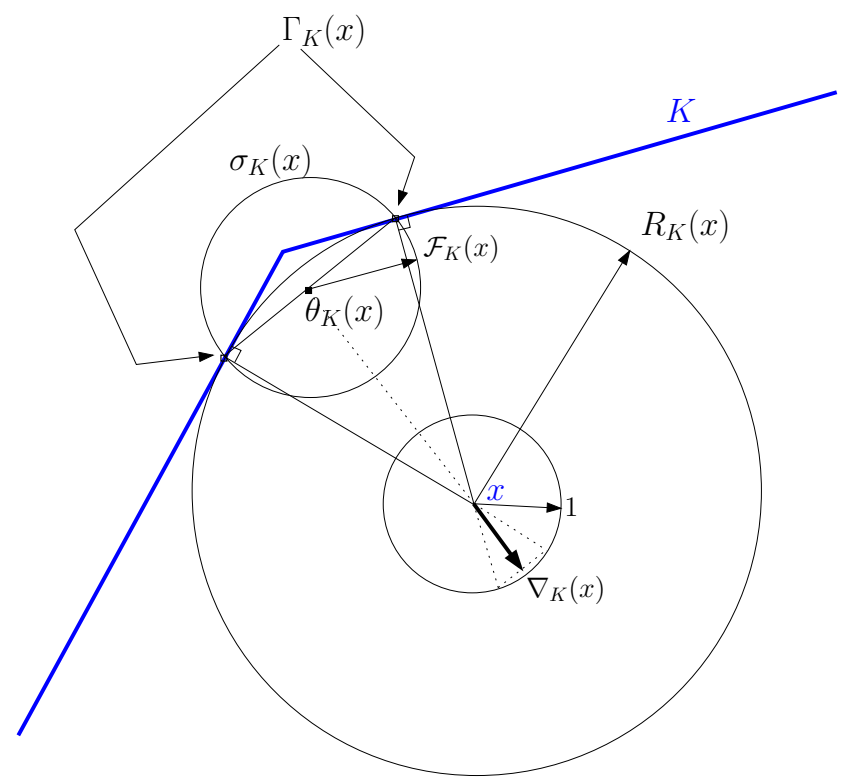

Figure 3: A 2-dimensional example with 2 closest points.

\subsection{The gradient and its flow.}

The distance function $R_{K}$ is not differentiable on $\mathcal{M}(K)$. However, it is possible [21] to define a generalized gradient function $\nabla_{K}: \mathbb{R}^{n} \rightarrow \mathbb{R}^{n}$ that coincides with the usual gradient of $R_{K}$ at points where $R_{K}$ is differentiable. For any point $x \in \mathbb{R}^{n} \backslash K$, we denote by $\Gamma_{K}(x)$ the set of points in $K$ closest to $x$ (figure 3 ):

$$
\Gamma_{K}(x)=\{y \in K \mid d(x, y)=d(x, K)\}
$$

Note that $\Gamma_{K}(x)$ is a non empty compact set. The function $x \mapsto \Gamma_{K}(x)$ is upper semi-continuous (cf. [21] and [13] p.29):

$$
\forall x, \forall r, \exists \alpha,\|y-x\| \leq \alpha \Rightarrow \Gamma_{K}(y) \subset \Gamma_{K}(x)_{r}
$$

There is a unique smallest closed ball $\sigma_{K}(x)$ enclosing $\Gamma_{K}(x)$ (cf. figure 3 ). We denote by $\theta_{K}(x)$ the center of $\sigma_{K}(x)$ and by $\mathcal{F}_{K}(x)$ its radius. $\theta_{K}(x)$ can equivalently be defined as the point on the convex hull of $\Gamma_{K}(x)$ nearest to $x$. For $x \in \mathbb{R}^{n} \backslash K$, the generalized gradient $\nabla_{K}(x)$ is defined as follows:

$$
\nabla_{K}(x)=\frac{x-\theta_{K}(x)}{R_{K}(x)}
$$

It is natural to set $\nabla_{K}(x)=0$ for $x \in K$. For $x \in \mathbb{R}^{n} \backslash K$, one has the following relation [21]:

$$
\left\|\nabla_{K}(x)\right\|^{2}=1-\frac{\mathcal{F}_{K}(x)^{2}}{R_{K}(x)^{2}}
$$


Equivalently, $\left\|\nabla_{K}(x)\right\|$ is the cosine of the (half) angle of the smallest cone with apex $x$ that contains $\Gamma_{K}(x)$. The map $x \mapsto\left\|\nabla_{K}(x)\right\|$ is lower semicontinuous [21]. Although $\nabla_{K}$ is not continuous, it is shown in [21] that Euler schemes using $\nabla_{K}$ converges uniformly, when the integration step decreases, toward a continuous flow $\mathfrak{C}: \mathbb{R}^{+} \times \mathbb{R}^{n} \rightarrow \mathbb{R}^{n}$. The integral line of this flow starting at a point $x \in \mathbb{R}^{n}$ can be parameterized by arc length $s \mapsto \mathfrak{C}(t(s), x)$. It is possible to express the value of $R_{K}$ at the point $\mathfrak{C}(t(l), x)$ by integration along the integral line with length $l$ downstream the point $x$ :

$$
R_{K}(\mathfrak{C}(t(l), x))=R_{K}(x)+\int_{0}^{l} \| \nabla_{K}(\mathfrak{C}(t(s), x) \| d s
$$

It is proven in [21] that the functions $\mathcal{F}_{K}$ and $R_{K}$ are increasing along the trajectories of the flow. In the particular case where $K$ is a finite set, various notions of flows related to this one have been independently introduced by H. Edelsbrunner [15], J. Giesen and al. [19] and R. Chaine [4] using Voronoï diagrams.

\subsection{Critical point theory for distance functions.}

The critical points of $R_{K}$ are defined as the points $x$ for which $\nabla_{K}(x)=0$. Equivalently, a point $x$ is a critical point if and only if it lies in the convex hull of $\Gamma_{K}(x)$. When $K$ is finite, this last definition means that critical points are precisely the intersections of Delaunay $k$-dimensional simplices with their dual $(n-k)$-dimensional Voronoï facets [19]. Note that this notion of critical point is the same as the one considered in the setting of non smooth analysis [13] and Riemannian geometry [12, 20]. The topology of the offsets $K_{r}$ of a compact set $K$ are closely related to the critical values of $R_{K}$ (i.e. the values of its distance function at critical points). The weak feature size of $K$, or wfs $(K)$, is defined as the infimum of the positive critical values of $R_{K}$. Equivalently it is the minimum distance between $K$ and the set of critical points of $R_{K}$. Notice that wfs $(K)$ may be equal to 0 . Nevertheless, $\mathrm{wfs}(K)$ is non zero for a large class of compact sets including polyhedrons and piecewise analytic sets (see $[8,9])$. The following result from [9] shows that wfs $(K)$ may be viewed as the "minimum size of the topological features" of the set $K$ :

Lemma 2.1 If $0<r, s<\operatorname{wfs}(K)$ then $K_{r}$ and $K_{s}$ are homeomorphic and even isotopic. The same holds for the complements of $K_{r}$ and $K_{s}$. The same also holds for the boundaries $\partial K_{r}$ and $\partial K_{s}$ that are topological $(n-1)$-dimensional manifolds.

Roughly speaking, two subspaces of $\mathbb{R}^{n}$ are isotopic if they can be deformed one into each other without tearing or self-intersection. For example, a circle and a trefoil knot are homeomorphic but not isotopic.

\subsection{The critical function and the $\mu$-reach}

The results of this paper rely strongly on the notions of critical function and $\mu$-reach, introduced in [7]. 

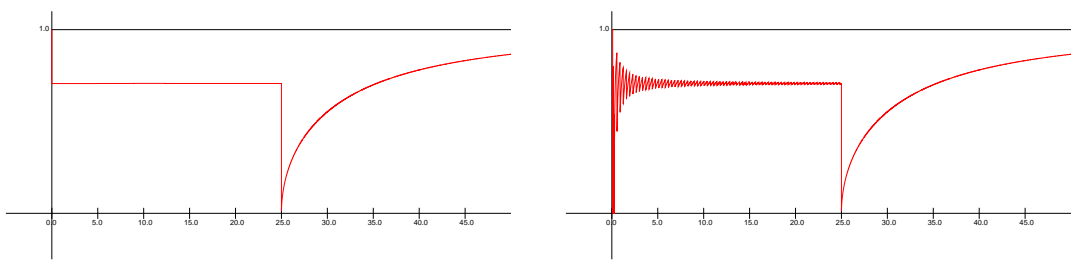

Figure 4: Critical function of a square embedded in $\mathbb{R}^{3}$ with side length 50 (left), and of a sampling of that square (right).

Definition 2.2 (critical function) Given a compact set $K \subset \mathbb{R}^{n}$, its critical function $\chi_{K}:(0,+\infty) \rightarrow$ $\mathbb{R}_{+}$is the real function defined by:

$$
\chi_{K}(d)=\inf _{R_{K}^{-1}(d)}\left\|\nabla_{K}\right\|
$$

Figure 4 shows the respective critical functions of a square in 3-space and of a sampling of it. We note that the infimum can be replaced by a minimum since $\left\|\nabla_{K}\right\|$ is lower semi-continuous and $R_{K}^{-1}(d)$ is compact. It also results from the compactness of $R_{K}^{-1}(d)$ that $d \mapsto \chi_{K}(d)$ is lower semi-continuous. The critical function is in some sense "stable" with respect to small (measured by Hausdorff distance) perturbations of a compact set, precisely [7]:

Theorem 2.3 (critical function stability theorem) Let $K$ and $K^{\prime}$ be two compact subsets of $\mathbb{R}^{n}$ and $d_{H}\left(K, K^{\prime}\right) \leq \varepsilon$. For all $d \geq 0$, we have:

$$
\inf \left\{\chi_{K^{\prime}}(u) \mid u \in I(d, \varepsilon)\right\} \leq \chi_{K}(d)+2 \sqrt{\frac{\varepsilon}{d}}
$$

where $I(d, \varepsilon)=\left[d-\varepsilon, d+2 \chi_{K}(d) \sqrt{\varepsilon d}+3 \varepsilon\right]$

Theorem 2.3 claim can be read as $\chi_{K}(d) \geq \inf \left\{\chi_{K^{\prime}}(u) \mid u \in I(d, \varepsilon)\right\}-2 \sqrt{\frac{\varepsilon}{d}}$ and says that the knowledge of a lower bound on the critical function of a compact set $K^{\prime}$ gives a lower bound on the critical function of "nearby" (for Hausdorff distance) compact sets $K$. In particular, if a set $K^{\prime}$ of measured points is known to lie within some Hausdorff distance of a physical object represented by the unknown compact set $K$, the critical function of $K^{\prime}$ gives, by theorem 2.3, a lower bound on the critical function of the partially known physical object $K$. Note that as explained in [7], starting from the Voronoi complex of the sample, the computation of the critical function of a finite sample is straightforward. This stability of the critical function with respect to small perturbations of the object in Hausdorff distance makes it realistic with respect to physical interactions - it does not relie on unmeasurable quantities - but also robust with respect to numerical computations because, by backward error analysis, the impact of rounding errors on the evaluation of the critical function can be controlled.

The $\mu$-reach of a compact set $K$ is the maximal offset value $d$ for which $\chi_{K}\left(d^{\prime}\right) \geq \mu$ for $d^{\prime}<d$. 


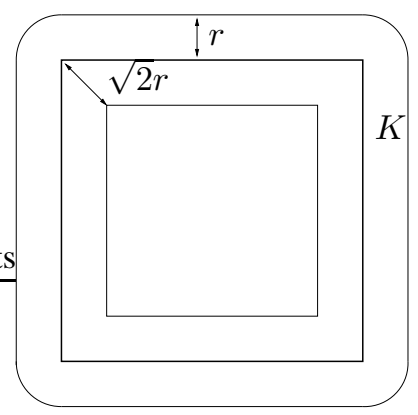

Figure 5: The boundary of the $r$-offset of a square $K$ in the plane is at Hausdorff distance $\sqrt{2} r$ of $K$. For $\mu=\frac{\sqrt{2}}{2}, r_{\mu}(K)>0$ and this example shows the tightness of the bound in theorem 3.2

Definition 2.4 ( $\mu$-reach) The $\mu$-reach $r_{\mu}(K)$ of a compact set $K \subset \mathbb{R}^{n}$ is defined by:

$$
r_{\mu}(K)=\inf \left\{d \mid \chi_{K}(d)<\mu\right\}
$$

We have that $r_{1}(K)$ coincides with the reach introduced by Federer [16]. As $\mu$ increases, $r_{\mu}(K)$ decreases and $\lim _{\mu \rightarrow 0^{+}} r_{\mu}(K) \leq \mathrm{wfs}(K)$. The inequality can be strict: for instance, if $K$ is the union of two tangent disks, then the $\mu$-reach of $K$ is 0 for all $\mu>0$, whereas $\operatorname{wfs}(K)=+\infty$. However, if the limit is positive, then equality holds. In any case, we have that $\lim _{\varepsilon \rightarrow 0^{+}}\left(\lim _{\mu \rightarrow 0^{+}} r_{\mu}\left(K^{\varepsilon}\right)\right)=$ $\operatorname{wfs}(K)$.

\section{Distance to offset boundary}

While the Hausdorff distance between a compact set $K$ and its $r$-offset is equal to $r$, usually the distance between $K$ and the boundary of $K_{r}$ is greater than $r$ (see figure 5). Nevertheless, we prove that this later distance can be bounded using the $\mu$-reach of $K$.

Lemma 3.1 Let $K$ be a compact set and let $\mu>0, r>0$ be such that $r<r_{\mu}(K)$. For any $x \in K_{r} \backslash K$, one has

$$
d\left(x, \partial K_{r}\right) \leq \frac{r-R_{K}(x)}{\mu} \leq \frac{r}{\mu}
$$

Proof. Let $t \rightarrow \mathcal{C}(t, x)$ be the trajectory of $\nabla_{K}$ issued from $x$. The lemma follows from the equation (2). Denoting

$$
s(t)=\int_{0}^{t}\left\|\nabla_{K}(\mathcal{C}(\tau, x))\right\|
$$

the arc length on the trajectory and $s \rightarrow t(s)$ its inverse, one has

$$
R_{K}(\mathcal{C}(t(s), x))=R_{K}(x)+\int_{0}^{s}\left\|\nabla_{K}(\mathcal{C}(t(\sigma), x))\right\| d \sigma
$$


Note that since $r<r_{\mu}(K)<\operatorname{wfs}(K)$, the trajectory of $\nabla_{K}$ issued from $x$ has to intersect $\partial K_{r}$ at some point $\mathcal{C}\left(t_{0}, x\right)$ and the distance from $x$ to $\partial K_{r}$ is bounded by the length of the part of the trajectory between 0 and $t_{0}$. The length of this part is $s_{0}=s\left(t_{0}\right)$. Since $R_{K}\left(\mathcal{C}\left(s\left(t_{0}\right), x\right)\right)=r$, and using that $\left\|\nabla_{K}\right\|$ is greater than $\mu$ in $K_{r}$, one has

$$
\begin{aligned}
r & =R_{K}(x)+\int_{0}^{s_{0}}\left\|\nabla_{K}(\mathcal{C}(t(\sigma), x))\right\| d \sigma \\
& \geq R_{K}(x)+s_{0} \mu
\end{aligned}
$$

which concludes the proof of the lemma.

Theorem 3.2 (Offset distance theorem) Let $K$ be a compact set and let $\mu>0, r>0$ be such that $r<r_{\mu}(K)$. Then $\partial K$ is at distance less than $\frac{r}{\mu}$ of the boundary $\partial K_{r}$ of $K_{r}$. More generally,

$$
d_{H}\left(\partial K, \partial K_{r}\right)<\frac{r}{\mu}
$$

Note that the example of figure 5 proves that the upper bound $\frac{r}{\mu}$ is tight.

PROOF. One just has to prove that for any $x \in \partial K$, there exits a point of $\partial K_{r}$ at distance no greater than $r / \mu$. Since $x \in \partial K$, there exists a sequence of points $x_{n} \in K_{r} \backslash K$ that converges to $x$. Applying previous lemma, one immediately deduces that

$$
d\left(x, \partial K_{r}\right)=\lim _{n \rightarrow \infty} d\left(x_{n}, \partial K_{r}\right) \leq \frac{r}{\mu}
$$

\section{Complements of offsets}

Let $K \subset \mathbb{R}^{n}$ be a compact set. For any $\left.\left.\mu \in\right] 0,1\right]$ denote by $r_{\mu}$ the $\mu$ - reach of $K$. In this section we study the critical function of the complements of the $r$-offsets $K_{r}$. It turns out that for $r<r_{\mu}$ it can be bounded from below by a function depending on $\mu$. Precisely, the complement of $K_{r}$ has positive reach bounded from below by $\mu r$.

\subsection{The critical function of offsets complements}

We prove that the complement $K_{r}^{c}$ of the offset $K_{r}$ has positive reach for any value $0<r<r_{\mu}$. Moreover, one gives a lower bound for the critical function of $K_{r}$.

Theorem 4.1 For $r \in\left(0, r_{\mu}\right)$, one has

$$
\operatorname{reach}\left(\overline{\mathrm{K}_{\mathrm{r}}^{\mathrm{c}}}\right) \geq \mu \mathrm{r}
$$


Moreover for any $d \in(\mu r, r)$,

$$
\chi_{\overline{K_{r}^{c}}}(d) \geq \frac{2 \mu r-d\left(1+\mu^{2}\right)}{d\left(1-\mu^{2}\right)}
$$

Note that the positivity of the reach of $K_{r}^{c}$ can be deduced from a result of J. Fu ([18], corollary 3.4) but without lower bound. It has also been proved for polyhedrons, still without lower bound, in an unpublished work by T. Ozawa ([23]).

A key argument in the proof of the theorem is the following elementary lemma.

Lemma 4.2 Let $x \in \mathbb{R}^{n}$. Then

$$
k_{x}(y)=\|y-x\|^{2}-R_{K}^{2}(y)
$$

is a convex function.

PROOF. One has

$$
\begin{aligned}
k_{x}(y) & =\|y-x\|^{2}-\inf _{z \in K}\|y-z\|^{2} \\
& =\sup _{z \in K}\left(\|y-x\|^{2}-\|y-z\|^{2}\right) \\
& =\sup _{z \in K}\left(2<(z-x), y>+\|x\|^{2}-\|z\|^{2}\right)
\end{aligned}
$$

So $k_{x}$ is the supremum of a family of linear functions and is thus convex.

PROOF. (Proof of 4.1)

Let $x \in\left(K_{r} \backslash K\right)$ and $\Gamma=\Gamma_{K_{r}^{c}}(x)$ be the set of nearest neighbors of $x$ in $\overline{K_{r}^{c}}$. To obtain the inequalities of the theorem, we compute and compare an upper and a lower bound of $R_{K}(y)$ where $y$ is the center of the ball of smallest radius $l \geq 0$ enclosing $\Gamma$.

First note that $y$ is contained in the convex hull of $\Gamma$. Otherwise, if $y^{\prime} \neq y$ is the nearest point of $y$ on $c o(\Gamma)$ then $\Gamma$ is on one side of the hyperplane passing through $y^{\prime}$ and orthogonal to $y^{\prime}-y$ (see figure 6). It follows that any smallest ball containing $\Gamma$ with center on the segment $\left[y, y^{\prime}\right]$ has smaller radius than the one with center $y$ : a contradiction.

As a consequence, there exists a finite set of points $y_{1}, \cdots y_{p} \in \Gamma$ such that $y=\lambda_{1} y_{1}+\cdots+\lambda_{p} y_{p}$ with $\lambda_{1}+\cdots+\lambda_{p}=1$ and $\lambda_{i}>0$ for any $i=1 \cdots p$.

Let $d=d\left(x, K_{r}^{c}\right)>0$ and let $\theta$ be the angle between $y-x$ and any vector joining $x$ to a point of the intersection of the two spheres $\mathbb{S}(x, d)$ and $\mathbb{S}(y, l)$ (see figure 7). Applying lemma 4.2, one obtains

$$
\begin{aligned}
k_{x}(y)=d^{2}-l^{2}-R_{K}(y)^{2} & \leq \sum_{i=1}^{p} \lambda_{i} k_{x}\left(y_{i}\right) \\
& =\sum_{i=1}^{p} \lambda_{i}\left(d^{2}-r^{2}\right) \\
& =d^{2}-r^{2}
\end{aligned}
$$




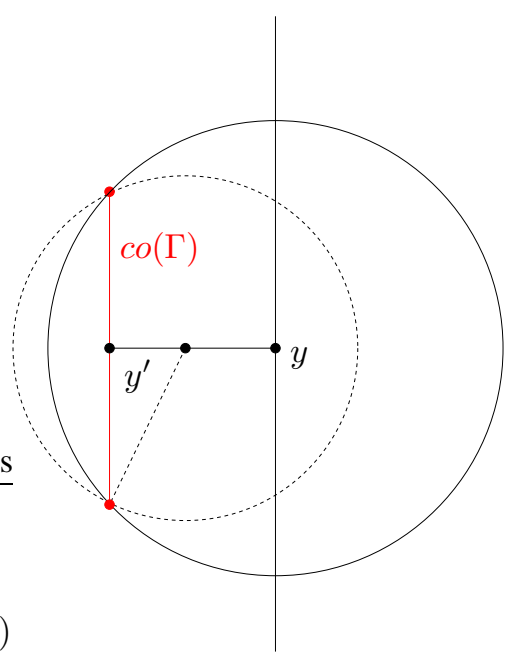

Figure 6:

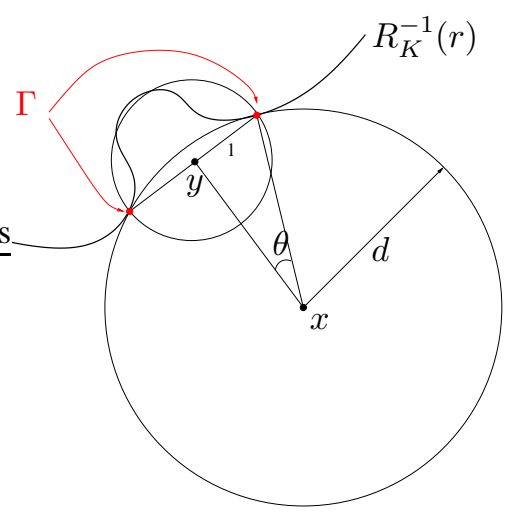

Figure 7: Proof of theorem 4.1

It follows that

$$
R_{K}(y)^{2} \geq r^{2}-l^{2}>0
$$

Note that the second inequality follows from the fact that $r>d \geq l$ since we only consider values $d<r$ in the statement of the theorem.

Now, let $t \rightarrow \mathcal{C}(t, y)$ be the trajectory of $\nabla_{K}$ issued from $y$ and let denote by $\tilde{y}$ the first intersection point of this trajectory with $\mathbb{S}(x, d)$. Let denote by $\tilde{l}$ the length of the trajectory $t \rightarrow \mathcal{C}(t, y)$ between $y$ and $\tilde{y}$. One has

$$
\tilde{l} \geq d(y, \mathbb{S}(x, d))=d(1-\cos \theta)
$$


Since $R_{K}$ is strictly increasing along the trajectory $t \rightarrow \mathcal{C}(t, y)$ and $R_{K}(y)>0$ (inequation (7)), the part of trajectory between $y$ and $\tilde{y}$ is contained in $R_{K}^{-1}((0, r])$. So $\left\|\nabla_{K}\right\|$ is at least $\mu$ along this part of trajectory which implies, in the same way as in the proof of lemma $3.1, R_{K}(\tilde{y}) \geq R_{K}(y)+\tilde{l} \mu$. Using that $R_{K}(\tilde{y}) \leq r$ one immediately obtains that

$$
R_{K}(y)^{2} \leq(r-\mu d(1-\cos \theta))^{2}
$$

Combining inequations (7) and (8), one obtains

$$
d^{2}\left(\mu^{2}(1-\cos \theta)^{2}+\sin ^{2} \theta\right)-2 \mu r(1-\cos \theta) d \geq 0
$$

When $\theta>0$, using that $d>0$ and $\sin ^{2} \theta=(1-\cos \theta)(1+\cos \theta)$ this boils down to

$$
d \geq \frac{2}{1+\mu^{2}+\left(1-\mu^{2}\right) \cos \theta} \mu r \geq \mu r
$$

So if $d<\mu r$ then $\theta=0$ and $\Gamma$ is reduced to a single point. This implies inequation (5). Now, if $d>\mu r$, the previous inequation is equivalent to

$$
\cos \theta \geq \frac{2 \mu r-d\left(1+\mu^{2}\right)}{d\left(1-\mu^{2}\right)}
$$

which implies the inequation (6) since $\cos \theta=\left\|\nabla_{K_{r}^{c}}(x)\right\|$.

Using that the map $x \rightarrow\left\|\nabla_{K}(x)\right\|$ semi-continuous, one can extend the theorem 4.1 to compact sets with positive wfs. If $K$ is a compact set such that wfs $(K)>0$, it may happen that $r_{\mu}(K)=0$ for any $\mu \in(0,1)$ (see figure 8). Nevertheless, since $\left\|\nabla_{K}\right\| \neq 0$ in the open set $R_{K}^{-1}((0, \mathrm{wfs}(K)))$, it follows from the lower semi-continuity of $\left\|\nabla_{K}\right\|$ that for any $0<\varepsilon<\frac{\operatorname{wfs}(K)}{2},\left\|\nabla_{K}\right\|$ reaches its upper lower bound $\mu$ on the compact set $R_{K}^{-1}([\varepsilon, \mathrm{wfs}(K)-\varepsilon])$, which is strictly positive. As a consequence, one has $\mu>0$ such that $r_{\mu}\left(K_{\varepsilon}\right) \geq \operatorname{wfs}(K)-2 \varepsilon>0$. Applying theorem 4.1 to $K_{\varepsilon}$ leads to the following result.

Theorem 4.3 Let $K$ be a compact set such that $\operatorname{wfs}(K)>0$. Let $0<\varepsilon<\operatorname{wfs}(K)$ and $\mu>0$ such that $r_{\mu}\left(K_{\varepsilon}\right) \neq 0$. For $r \in\left(0, r_{\mu}\left(K_{\varepsilon}\right)\right)$ and $d \in(\mu r, r)$ one has

$$
\operatorname{reach}\left(\overline{\mathrm{K}_{\varepsilon+\mathrm{r}}^{\mathrm{c}}}\right) \geq \mu \mathrm{r} \text { and } \chi_{\left(\overline{\mathrm{K}_{\varepsilon+\mathrm{r}}^{\mathrm{c}}}\right)}(\mathrm{d}) \geq \frac{2 \mu \mathrm{r}-\mathrm{d}\left(1+\mu^{2}\right)}{\mathrm{d}\left(1-\mu^{2}\right)}
$$

In particular, the complement of any $r$-offset of $K, 0<r<\mathrm{wfs}(K)$, has positive reach.

\subsection{Tightness of the bound}

The lower bound for the reach given in theorem 4.1 is tight. Let $\alpha \in(0, \pi / 2)$ and let $P=$ $(0, \cos \alpha, \sin \alpha) \in \mathbb{R}^{3}$ and $Q=(0, \cos \alpha,-\sin \alpha) \in \mathbb{R}^{3}$. Consider the compact set $K \subset \mathbb{R}^{3}$ defined as the union of the two segments joining the origin $O=(0,0,0)$ to $P$ and $Q$ respectively (see figure 9). An easy computation shows that $r_{\mu}=0$ for $\mu>\sin \alpha$ and $r_{\mu}=+\infty$ for $\mu \leq \sin \alpha$. For any $0<r<\tan \alpha$ the boundary of $K_{r}$ intersects the plane $\{z=0\}$ along a piece of ellipse in a neighborhood of the point $P_{\alpha}=\left(0, \frac{r}{\sin \alpha}, 0\right)$ (see figure 9). Moreover the radius of curvature of this ellipse at $P_{\alpha}$ is equal to $r \sin \alpha$. It follows that the reach of $K_{r}^{c}$ is at $\operatorname{most} r \sin \alpha=r \mu$ for $\mu=\sin \alpha$. 


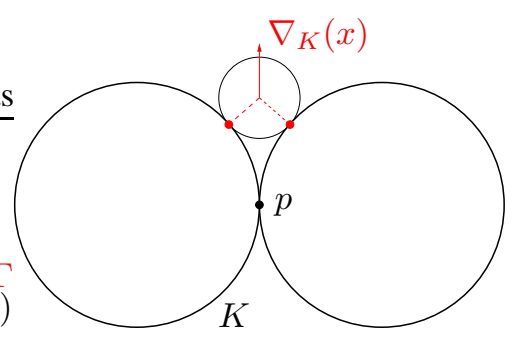

Figure 8: The union of two tangent discs in the plane has a positive wfs and zero $\mu$-reach for any $\mu \in(0,1)$ : if $p$ is the intersection point of the two discs, then $\nabla_{K}(x)$ tends to 0 as $x \in K^{c}$ tends to $p$

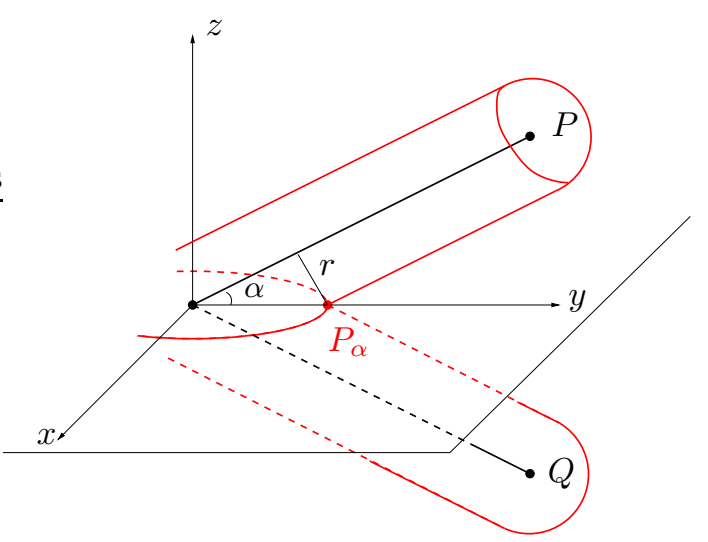

Figure 9: Tightness of the bound 


\section{Double offsets}

Let $K \subset \mathbb{R}^{n}$ be a compact set. For $0<d<r$, the $(r, d)$-double offset $K_{r, d}$ of $K$ is the set of points that are at distance $d$ of $K_{r}^{c}$ :

$$
K_{r, d}=\left\{x \in \mathbb{R}^{n}: d\left(x, K_{r}^{c}\right)=d\right\}
$$

\subsection{Smoothness of double offsets}

Using a result of Federer ([16], theorem 4.8) stating that the distance function to a closed set $A$ with positive reach $r_{1}$ is differentiable with non-zero Lipschitz gradient on the complement of the closure of the medial axis of $A$, one obtains the following result.

Theorem 5.1 (Double offset theorem) If $r<r_{\mu}$ for some value $\mu>0$ and if $d<\mu r$ then $K_{r, d}$ is a smooth $\mathcal{C}^{1,1}$-hypersurface. Moreover,

$$
\operatorname{reach}\left(\mathrm{K}_{\mathrm{r}, \mathrm{d}}\right) \geq \min (\mathrm{d}, \mu \mathrm{r}-\mathrm{d})
$$

which implies that the smallest of the principal radii of curvature at any point of $K_{r, d}$ is at least $\min (d, \mu r-d)$.

PROOF. Let $R_{r}:=R_{K_{r}^{c}}$ be the distance function to $K_{r}^{c}$. Federer's result ([16], theorem 4.8) implies that $R_{r}$ is a $\mathcal{C}^{1,1}$ function without critical point (in the sense of classical differential calculus) in the open set $R_{r}^{-1}((0, \mu r))$. Since $K_{r, d}$ is a level set of $R_{r}$ contained in this open set, the first part of the theorem follows immediately from the implicit function theorem. The second part is an immediate consequence of inequation (5).

\subsection{Distance to double offset}

As in the case of offsets, one gives an upper bound on the Hausdorff distance between $K_{r, d}$ and $\partial K$.

Proposition 5.1 If $r<r_{\mu}$ for some value $\mu>0$ and if $d<\mu r$ then

$$
d_{H}\left(K_{r, d}, \partial K\right) \leq \frac{r}{\mu}-d
$$

PROOF. Let $x \in K_{r, d}$ and let $t \rightarrow \mathcal{C}(t, x)$ be the trajectory of $\nabla_{K}$ issued from $x$. Since $\left\|\nabla_{K}\right\|$ is at least $\mu>0$ in $K_{r}$, this trajectory intersects $R_{K}^{-1}(r)$ the boundary of $K_{r}^{c}$ in some point $x^{\prime}$. Since $x$ is at distance $d$ from $R_{K}^{-1}(r)$, the lenght $l$ of the trajectory between $x$ and $x^{\prime}$ is at least $d$. So, using equation (2) one obtains

$$
\begin{aligned}
r=R_{K}\left(x^{\prime}\right) & =R_{K}(x)+\int_{0}^{l} \| \nabla_{K}(\mathfrak{C}(t(s), x) \| d s \\
& \geq R_{K}(x)+\int_{0}^{d} \| \nabla_{K}(\mathfrak{C}(t(s), x) \| d s \\
& \geq R_{K}(x)+\mu d
\end{aligned}
$$




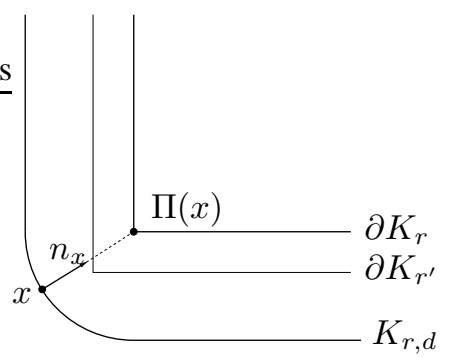

Figure 10:

It follows that $K_{r, d} \subset K_{r-\mu d}$. Now, the offset distance theorem 3.2 applied to $K_{r-\mu d}$ gives the result.

The bound of previous proposition is tight: consider the example of section 4.2, for $\mu=\sin \alpha$. The point $m=\left(0, \frac{r}{\sin \alpha}-d, 0\right)$ is on $K_{r, d}$ and is at Haussdorf distance $\left(\frac{r}{\sin \alpha}-d\right) \cos \alpha$ from $K$. But the difference between this distance and the bound of the proposition is $\left(\frac{r}{\mu}-d\right)(1-\cos \alpha)$. This difference can be made as small as one wants by choosing $\alpha$ as small as necessary (note that $\mu$ also changes with the choice of $\alpha$ ).

\subsection{Topology of double offsets}

In this section we prove that smoothing offsets by double-offset does not change the topology. More precisely, we prove that boundaries of offsets and double-offsets are isotopic. We also give a stronger result in dimension 3 that relates the topology of a topological surface $S$ to the topology of its offsets. Let $K$ be a compact set and let $\mu>0$ be such that $r_{\mu}>0$. First recall from lemma 2.1 that for any values $r, r^{\prime}<\operatorname{wfs}(K), \partial K_{r}$ and $\partial K_{r^{\prime}}$ are isotopic hypersurfaces.

Theorem 5.2 (Offsets isotopy theorem) For $r<r_{\mu}$ and $0<d<\mu r, \partial K_{r}$ and $K_{r, d}$ are isotopic hypersurfaces.

Proof. Let $r$ and $d$ be as in the theorem. Since $K_{r, d}$ is a smooth hypersurface, for each point $x \in K_{r, d}$ one denotes by $n_{x}$ the unit vector normal to $K_{r, d}$ at $x$ that points in the direction of $\Pi(x)$ the nearest point from $x$ on $\partial K_{r}$ (see figure 10). Remark that $\Pi(x)$ is the first point of intersection of the half-line issued from $x$ and directed by $n_{x}$ and that $d(x, \Pi(x))=d$. Moreover, since $K_{r, d}$ is smooth, the projection $p$ to $K_{r, d}$ along the normals of $K_{r, d}$ is continuous and well-defined on the "band"

$$
B=\bigcup_{x \in K_{r, d}}[x, \Pi(x))
$$

It follows from the proof of proposition 5.1 that $R_{K}(x) \leq r-\mu d$ for any $x \in K_{r, d}$. As a consequence, for $r^{\prime}=r-\mu d, \partial K_{r^{\prime}} \subset B$. and the restriction $p^{\prime}$ of $p$ to $\partial K_{r^{\prime}}$ is a continuous map. Moreover, since for any $x \in K_{r, d}, R_{K}(x) \leq r-\mu d$ and $R_{K}(\Pi(x))=r$ the map $p^{\prime}$ is surjective. The injectivity of $p^{\prime}$ follows from the following lemma. 


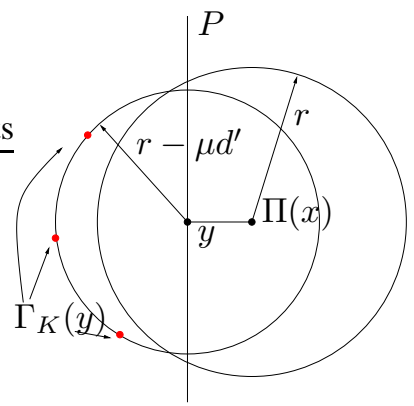

Figure 11:

Lemma 5.3 Let $x \in K_{r, d}$. The restriction of $R_{K}$ to $[x, \Pi(x))$ is strictly increasing. In particular, the segment $[x, \Pi(x)]$ intersects $\partial K_{r^{\prime}}$ in exactly one point.

Proof. Let $y \in[x, \Pi(x))$ and let $d^{\prime}=d(y, \Pi(x))=d\left(y, \partial K_{r}\right)$. Remark that $y \in K_{r, d^{\prime}}$ and thus $R_{K}(y) \leq r-\mu d^{\prime}$ as in the proof of proposition 5.1. Using that $\mathbb{B}(\Pi(x), r) \cap K=\emptyset$, one deduces that $\Gamma_{K}(y) \subset \mathbb{S}\left(y, r-\mu d^{\prime}\right) \backslash \mathbb{B}(\Pi(x), r)$. Let $P$ be the hyperplane passing through $y$ and orthogonal to $[x, \Pi(x))$ (see figure 11). $\Gamma_{K}(y)$ is contained in the half-space defined by $P$ if and only if

$$
\left(r-\mu d^{\prime}\right)^{2}<r^{2}-d^{\prime 2}
$$

that is equivalent to

$$
d^{\prime}<\frac{2 \mu}{\mu^{2}+1} r
$$

Since $d^{\prime} \leq d \leq \mu r$, this last inequation is satisfied. It then follows from the upper semi-continuity of the map $x \rightarrow \Gamma_{K}(x)$ that there exists $\delta, \alpha>0$ such that for any $t \in(-\delta ;+\delta), R_{K}\left(y+t n_{x}\right) \geq$ $R_{K}(y)+\alpha t$. This last inequality proves the lemma.

The previous lemma immediately implies that the projection $p^{\prime}$ is injective and thus bijective. Since it is a continuous bijection between compact sets, this is an homeomorphism between $\partial K_{r^{\prime}}$ and $K_{r, d}$. If one denotes by $\phi: K_{r, d} \rightarrow \partial K_{r^{\prime}}$ its inverse, the map $F: K_{r, d} \times[0,1] \rightarrow \mathbb{R}^{n}$ defined by $F(x, t)=x+t(\phi(x)-x)$ defines an isotopy between $\partial K_{r^{\prime}}$ and $K_{r, d}$.

The 3-dimensional case. We now adress the particular case of surfaces embedded in $\mathbb{R}^{3}$. Recall that a topological surface $S$ embedded in $\mathbb{R}^{3}$ is bicollar if there exists a neighborhood $M$ of $S$ that is a topological thickening of $S$, i.e. there exists a homeomorphism $\Phi: S \times[0,1] \rightarrow M$ satisfying $\Phi(S \times\{1 / 2\})=S \subset M$. Notice that such a topological thickening has two boundary components $\phi(S \times\{0\})$ and $\phi(S \times\{1\})$. Moreover, most of the compact surfaces encountered in practical applications are bicollar: for example, smooth surfaces, polyhedral surfaces, piecewise analytic surfaces,... are bicollar. 
Theorem 5.4 Let $S \subset \mathbb{R}^{3}$ be a bicollar surface such that $\operatorname{wfs}(S)>0$. Then for any $r<\operatorname{wfs}(S), S$ is isotopic to one of the components of $\partial S_{r}$.

This theorem combined with the previous one implies that a compact bicollar surface in $\mathbb{R}^{3}$ with positive wfs is isotopic to its $(r, d)$-double-offsets for $r<r_{\mu}$ and $d<\mu r$. The proof of theorem 5.4 is based upon rather different technics than the ones used in this paper. It is a consequence of the following result.

Theorem 5.5 ([6]) Let $S$ and $\tilde{S}$ be two compact topological surfaces embedded in $\mathbb{R}^{3}$ such that 1. $\tilde{S}$ is included in a topological thickening $M$ of $S$.

2. $S$ is included in a topological thickening $\tilde{M}$ of $\tilde{S}$.

3. $\tilde{S}$ separates the sides of $M$

4. $S$ separates the sides of $\tilde{M}$

Then $S$ and $\tilde{S}$ are isotopic in $M$ and in $\tilde{M}$.

Proof. Let $M$ be a topological thickening of $K$ and let $\Phi: S \times[0,1] \rightarrow M$ be the corresponding homeomorphism. The surface $S_{\delta}=\phi(S \times\{\delta\})$ is isotopic to $S$. For any values $0<r_{1}<r<r_{2}<$ wfs $(S)$, the "band" $B\left(r_{1}, r_{2}\right)=R_{K}^{-1}\left(\left[r_{1}, r_{2}\right]\right)$ is a a topological thickening of $\partial K_{r}$. If one chooses $r_{1}$ and $\delta$ sufficiently small, $S_{\delta}$ is contained in $B\left(r_{1}, r_{2}\right)$ and separates its sides. If one chooses $r_{2}$ sufficiently small, $\partial K_{r}$ is contained in $M$ and separates its sides. It sufficies to apply theorem 5.5 to conclude the proof.

\section{6 conclusion}

We have given, for the first time, a tight condition for the smoothness of double offsets. Our condition relies on the notion of critical functions or $\mu$-reach which are robusts and can be estimated from Hausdorff approximations such as finite point samples.

A natural extension of this work would be to consider "offsets with variable radius", because the simplification of realistic objects may require to adapt the offset distance to local object geometry and thus replace the uniform notion of $\mu$ - reach by a local function.

Another natural issue is the question of tightness in dimension 2. We believe that, in the conditions of theorem 4.1 but when the dimension of the ambient space is 2 , one has merely reach $\left(\overline{\mathrm{K}_{\mathrm{r}}^{\mathrm{c}}}\right) \geq \mathrm{r}$ instead of $\operatorname{reach}\left(\overline{\mathrm{K}_{\mathrm{r}}^{\mathrm{r}}}\right) \geq \mu \mathrm{r}$.

\section{References}

[1] N Amenta, TJ Peters, A Russell Computational Topology: Ambient Isotopic Approximation of 2-Manifolds Theoretical Computer Science, 2003

[2] L. Andersson and T. Peters and N. Stewart Self-intersection of composite curves and surfaces Computer Aided Geometric Design, (15), No. 5, 507 - 527, 1998. 
[3] L. Andersson and T. Peters and N. Stewart Equivalence Of Topological Form For Curvilinear Geometric Objects International Journal Computational Geometry and Applications, 10(6):609-622,2000.

[4] R. Chaine, A Geometric Convection Approach of 3-D Reconstruction In 1st Symposium on Geometry Processing, pp. 218-229, 2003

[5] F. Chazal, Quelques contributions en approximation géométrique et topologique, en analyse dynamique d'algorithmes et à l'étude des feuilletages non spiralants, Habilitation à Diriger des Recherches, Université de Bourgogne, 2005.

[6] F. Chazal, D. Cohen-Steiner, A condition for isotopic approximation, proc. ACM Symp. Solid Modeling and Applications 2004.

[7] F. Chazal, D. Cohen-Steiner, A. Lieutier, A Sampling Theory for Compacts in Euclidean Space, Proceedings of the 22nd ACM Symposium on Computational Geometry 2006

[8] F. Chazal, A. Lieutier, The $\lambda$-medial axis In Graphical Models (GMOD),vol. 67 (4) pp. 304-331, 2005

[9] F. Chazal, A. Lieutier, Weak feature size and persistent homology: computing homology of solids in $\mathbb{R}^{n}$ from noisy data samples, 21st Symposium on Computational geometry (2005) pp. 255-262

[10] F. Chazal, A. Lieutier, Weak feature size and persistent homology: computing homology of solids in $\mathbb{R}^{n}$ from noisy data samples preprint of the Institut Mathématiques de Bourgogne. Available at http://math.ubourgogne.fr/topologie/chazal/publications.htm

[11] David Cohen-Steiner, Herbert Edelsbrunner and John Harer Stability of Persistence Diagrams, 21st Symposium on Computational geometry (2005)

[12] J. Cheeger, Critical Points of Distance Functions and Applications to Geometry, Geometric Topology: recent developments,Montecatini Terme, 1990, Springer Lecture Notes, 1504 (1991), 1-38.

[13] F.H. Clarke, Optimization and NonSmooth Analysis, Wiley-Interscience, New-York, 1983.

[14] O. Devillers and P. Guigue, Inner and Outer Rounding of Boolean Operations on Lattice Polygonal Regions, Comp. Geometry Theory and Applications, 33:3-17, 2005.

[15] H. Edelsbrunner. Surface reconstruction by wrapping fi nite point sets in space, in Ricky Pollack and Eli Goodman Festschrift ed. B. Aronov, S. Basu, J. Pach and M. Sharir.Springer-Verlag, 379-404

[16] H. Federer, Curvature measures, Trans. Amer. Math. Soc. Vol. 93, p 418 (1959).

[17] S. Fortune, Vertex-rounding a three-dimensional polyhedral subdivision Dicrete Computational Geometry, 22(4):593-618, 1999

[18] J.H.G. Fu, Tubular Neighborhoods in Euclidean Spaces, Duke Math. Journal, Vol. 52, No. 4 (1995).

[19] J. Giesen and M. John, The Flow Complex: A Data Structure for Geometric Modeling. Proc. 14th Annual ACM-SIAM Symposium on Discrete Algorithms (SODA), (2003) 285-294.

[20] K. Grove, Critical Point Theory for Distance Functions, Proc. of Symposia in Pure Mathematics, Vol. 54 (1993), Part 3.

[21] A. Lieutier, Any open bounded subset of $\mathbb{R}^{n}$ has the same homotopy type as its medial axis, ComputerAided Design, 36(2004) 1029-1046, Elsevier.

[22] J. Serra and G. Matheron. Image Analysis and Mathematical Morphology, Volume 2: Theoretical Advances, Edited by Jean Serra Academic Press, 1988.

[23] T. Ozawa, Approximation of a PL-hypersurface by $\mathcal{C}^{1}$-hypersurfaces, private communication. 
[24] J. Rossignac, Blending and Offsetting Solid Models, PhD Dissertation, Electrical Engineering Department, University of Rochester, NY, June 1985.

[25] J. Rossignac and A. Requicha. Constant-radius blending in solid modeling. ASME Computers In Mechanical Engineering (CIME), 3:65-73, 1984.

[26] J. Rossignac and A. Requicha. Offsetting operations in solid modelling. Computer-Aided Geometric Design, 3:129-148, 1986. 


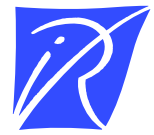

Unité de recherche INRIA Futurs

Parc Club Orsay Université - ZAC des Vignes

4, rue Jacques Monod - 91893 ORSAY Cedex (France)

Unité de recherche INRIA Lorraine : LORIA, Technopôle de Nancy-Brabois - Campus scientifi que 615, rue du Jardin Botanique - BP 101 - 54602 Villers-lès-Nancy Cedex (France)

Unité de recherche INRIA Rennes : IRISA, Campus universitaire de Beaulieu - 35042 Rennes Cedex (France)

Unité de recherche INRIA Rhône-Alpes : 655, avenue de l'Europe - 38334 Montbonnot Saint-Ismier (France) Unité de recherche INRIA Rocquencourt : Domaine de Voluceau - Rocquencourt - BP 105 - 78153 Le Chesnay Cedex (France)

Unité de recherche INRIA Sophia Antipolis : 2004, route des Lucioles - BP 93 - 06902 Sophia Antipolis Cedex (France) 